OPEN ACCESS

Edited by:

Chang Zou,

Jinan University, China

Reviewed by:

Saisha Nalawade,

Baylor College of Medicine,

United States

Nasser Pouladi,

Azarbaijan Shahid Madani University,

Iran

*Correspondence:

Xiaolan Guo

alan5200@hotmail.com

†These authors have contributed equally to this work

Specialty section:

This article was submitted to Molecular and Cellular Oncology,

a section of the journal

Frontiers in Cell and Developmental

Biology

Received: 24 June 2021 Accepted: 30 September 2021 Published: 27 October 2021

Citation:

Yao L, Zhong X, Huang G, Ma Q,

$X u L$, Xiao $H$ and Guo X (2021)

Investigation on the Potential

Correlation Between TP53

and Esophageal Cancer.

Front. Cell Dev. Biol. 9:730337.

doi: 10.3389/fcell.2021.730337

\section{Investigation on the Potential Correlation Between TP53 and Esophageal Cancer}

\author{
Lihua Yao ${ }^{1 \dagger}$, Xiaowu Zhong ${ }^{2 \dagger}$, Guangcheng Huang ${ }^{1}$, Qiang Ma', Lei Xu' ${ }^{2}$, Hong Xiao' and \\ Xiaolan Guo ${ }^{\text {* }}$ \\ ${ }^{1}$ Department of Clinical Laboratory, Affiliated Hospital of North Sichuan Medical College, Nanchong, China, ${ }^{2}$ Department \\ of Laboratory Medicine, North Sichuan Medical College, Nanchong, China
}

Background: TP53 family members play an indispensable role in various human cancers, while the gene expression profiles, prognostic value, and potential mechanism in esophageal cancer (ESCA) are yet unclear.

Methods: The expression and roles of TP53 family members in ESCA were investigated using the Cancer Genome Atlas (TCGA), Tumor Immune Estimation Resource (TIMER), Kaplan-Meier plotter, gene set enrichment analysis (GSEA), and UALCAN databases. The expression of TP53 between ESCA and the corresponding adjacent tissues was validated using QRT-PCR. Furthermore, the effects of TP53 on esophageal squamous cell carcinoma (ESCC) cell migration and proliferation were examined using the Transwell assay, scratch test, and crystal violet assay. The correlation between TP53 and mTOR pathways was evaluated by Western blotting.

Results: This study showed a correlation between high mRNA expression of TP53 members (TP53, TP63, and TP73) and clinical cancer stages and nodal metastasis status in ESCA patients. Moreover, the expression of TP53 was significantly associated with the overall survival (OS) of ESCA patients. Additional experiments verified that the mRNA of TP53 was upregulated in ESCC patients. Moreover, the downregulated expression of TP53 significantly retarded ESCC cell migration and proliferation and might activate the mTOR signaling pathway and inhibit TP53-dependent autophagy.

Conclusion: TP53 has a prognostic value in ESCA and may be a leading factor in promoting ESCA pathogenesis.

Keywords: esophageal cancer, expression, prognosis, TP53, bioinformatics

\section{INTRODUCTION}

Esophageal cancer (ESCA) is the leading cause of digestive system cancer mortality (Global Burden of Disease Cancer Collaboration et al., 2015). About 300,000 people die of esophageal cancer worldwide, with about 460,000 new cases every year (Ferlay et al., 2013). It is histologically defined by both esophageal squamous cell carcinoma (ESCC) and esophageal adenocarcinoma (EAC). Approximately 90\% of ESCA cases worldwide are ESCC (Arnold et al., 2015) and the major histological type is in East Asian countries like China (Lin et al., 2013), while the morbidity and mortality of ESCC are ranked fifth and fourth, respectively (Chen W. et al., 2015). The etiology of ESCA is associated with ethnicity, genetics, and dietary habit (Abnet et al., 2018). It is characterized by epigenetic abnormalities and disorders in the signaling pathways, which is consistent with other 
cancers (Chen J. et al., 2015). The 5-year overall survival (OS) rate of ESCA patients after the operation has greatly improved over the last couple of decades, while the OS and the prognosis are still poor, and approximately $20 \%$ are due to late diagnosis (Dubecz et al., 2012). The ESCA morbidity concealment, rapid progression, and lack of effective means for the early diagnosis are the main causes of high mortality (Chen et al., 2007). Thus, identifying specific and sensitive biomarkers and understanding the pathogenetic mechanisms underlying ESCA are valuable for clinicians to choose appropriate treatments to improve the survival rate of patients.

The TP53 family of transcription factors, including TP53, TP63, and TP73, plays key roles in biological and pathological processes of cancer and neural development (Agostini et al., 2018). These three proteins have a very similar domain organization and, hence, overlapping functions. These proteins also have unique functions: TP53 regulates the stress response to suppress tumors (Levine, 2020b), TP63 is essential for ectoderm development (Santos-Pereira et al., 2019), and TP73 regulates both stress response and development (Levrero et al., 2000). TP53 is a tumor suppressor gene associated with neoplastic disease (Bykov et al., 2018), while TP53 gene mutations occur in about half of human cancers (Kandoth et al., 2013). Approximately $90 \%$ of ESCAs harbor TP53 mutations (Gao et al., 2014), indicating that TP53 has a significant role in ESCA. The primary function of TP53 is as a transcription factor that protects the cells from various stresses, including autophagy, apoptosis, and senescence (Lane, 1992; Vousden and Prives, 2009; Ryan, 2011; Bieging et al., 2014). TP53 gene mutations cause a loss of the tumor suppressor gene and also contribute to tumorigenesis, such as increased genomic instability and cell proliferation, enhanced invasion and metastasis, drug resistance, and inhibition of apoptosis (Oren and Rotter, 2010). However, only a few studies have assessed the correlation between TP53 family members and ESCA. Therefore, a comprehensive analysis of the role of TP53 family in ESCA was imperative.

In the current study, we examined the mRNA expression of TP53 family members on ESCA using TIMER and UALACN database. Next, we analyzed the correlation between clinicopathological features and prognostic values of TP53 family members in ESCA. Additionally, we verified TP53 mRNA expression and clinicopathological characteristics in ESCC tissue. Subsequently, downregulated TP53 significantly inhibits ESCC cell proliferation and migration via mTOR signaling pathway and TP53-dependent autophagy. This study showed the prognostic value and potential biological function of TP53 family members in ESCA, and hence, could be considered a diagnostic biomarker and a promising therapeutic target in ESCC.

\section{MATERIALS AND METHODS}

\section{Expression Analysis of TP53 Family Members}

Tumor Immune Estimation Resource (TIMER) is a public database that uses microarrays for estimating the gene expression. UALCAN is a cancer information analysis platform consisting of a transcriptional expression database, and the corresponding clinical information is based on the TCGA cancer data. In this study, the transcriptional expression data of TP53 family genes were obtained from the TIMER and UALCAN databases.

\section{Clinicopathological Analysis of TP53 Family Members in Esophageal Cancer}

The correlation between the expression of TP53 family genes and clinicopathological parameters, including cancer stage, lymph node metastasis, tumor grade, and tumor histology was analyzed using the UALCAN database.

\section{Immune Infiltration Analysis}

Furthermore, the immune infiltration of TP53 family members in ESCA was evaluated using TIMER. The scatterplots of TP53 family members were generated to explore the correlation between the gene expression level and the abundance of immune cell infiltration using Spearman's correlation analysis.

\section{Survival Analysis}

In this study, we used the Kaplan-Meier plot to analyze the prognostic value of mRNA expression of TP53 family members in ESCA. Patients with ESCC and EAC were categorized into highand low-expression groups, according to the median values of mRNA expression.

\section{Populations and Ethics Statement}

This study was approved by the Medical Ethics Committee of the Affiliated Hospital of North Sichuan Medical College; the hospital is located in Nanchong, Sichuan Province, China. The patients provided their written informed consent to participate in this study. Between July 2015 and March 2016, cancer tissues and paracarcinoma normal tissues samples were obtained from 65 patients with ESCC who had undergone esophagectomy at the Department of Cardiothoracic Surgery, without preoperative chemotherapy or radiation. All patients were pathologically diagnosed with ESCC using surgical specimens and biopsies. All the tissue samples were surgically isolated within $30 \mathrm{~min}$ to excise an appropriate amount of tumor and normal tissue, soaked in RNAlater solution (Ambion, Carlsbad, CA, United States), and stored at $-80^{\circ} \mathrm{C}$ until further processing.

\section{Cell Culture}

Human normal esophageal epithelial cell line (HET-1A) was purchased from American Type Culture Collection (ATCC, Manassas, VA, United States), and human ESCC cell lines (TE1 and Kyse150) were obtained from the Cell Bank of Shanghai Institute of Cell Biology (Chinese Academy of Medical Sciences, Shanghai, China). All the cells were maintained in RPMI-1640 medium (Gibco, Grand Island, NY, United States) containing $10 \%$ fetal bovine serum (FBS, Gibco) and $1 \%$ penicillinstreptomycin (Invitrogen, Waltham, MA, United States) at $37^{\circ} \mathrm{C}$ with $5 \% \mathrm{CO}_{2}$ in the humidified incubator.

\section{RNA Extraction and Reverse Transcription}

The total RNA was extracted from ESCC tissues and cell lines using the TRIzol reagent (Ambion) according to 
the manufacturer's instructions. Reverse transcription was performed using the Transcriptor First Strand cDNA Synthesis Kit (Roche Diagnostics, Indianapolis, IN, United States), and subsequently, the cDNA was collected and stored at $-80^{\circ} \mathrm{C}$.

\section{Quantitative Real-Time PCR}

Quantitative Real-Time PCR was detected by the Lightcycler 480 Real-time PCR system (Roche, Mannheim, Germany) under the following conditions: $95^{\circ} \mathrm{C}$ for $5 \mathrm{~min}$, followed by 40 cycles of $10 \mathrm{~s}$ at $95^{\circ} \mathrm{C}, 15 \mathrm{~s}$ at $62^{\circ} \mathrm{C}, 72^{\circ} \mathrm{C}$ for $10 \mathrm{~s}$, $65^{\circ} \mathrm{C}$ for $60 \mathrm{~s}$, and $37^{\circ} \mathrm{C}$ for $30 \mathrm{~s}$. For TP53 mRNA detection, forward primer 5' $5^{\prime}$-CCAGGGCAGCTACGGTTTC- $3^{\prime}$ and reverse primer $5^{\prime}$-CTCCGTCATGTGCTGTGACTG-3'. $\beta$-Actin was used as an internal control: forward primer $5^{\prime}$-GGACTTCGAGCAAGAGATGG-3' and reverse primer 5'-AGCACTGTGTTGGCGTACAG-3'. Each experiment was performed in triplicate, and the relative expression of mRNA was normalized to the endogenous expression level of $\beta$-actin through the $2^{-\Delta \Delta} \mathrm{Ct}$ method.

\section{Plasmid and Stable Cell Transfection}

Kyse150 and TE1 cells were selected for further functional research. Plasmids encoding knockdown TP53 (sh-TP53) and corresponding controls (sh-NC) were purchased from Hanbio (Hanbio, Shanghai, China). According to the manufacturer's protocol, 50-60\% of confluent ESCC cells were transfected with plasmids using Lipofectamine 2000 reagent (Invitrogen, Waltham, MA, United States). The stably transfected cells were screened in the presence of $2 \mu \mathrm{g} / \mathrm{ml}$ puromycin for 14 days (Sigma, Chicago, IL, United States).

\section{Western Blot}

Total protein was extracted from tissues and cell pellet lysis, and the concentration of protein in the lysate was determined using the Pierce BCA Protein Assay Kit (Thermo Fisher Scientific, Rockford, IL, United States). Proteins were separated by $12 \%$ sodium dodecyl sulfate polyacrylamide gel electrophoresis (SDSPAGE) and transferred to polyvinylidene difluoride (PVDF) membranes (Millipore; Merck KGaA, Darmstadt, Germany). After blocking with 5\% skimmed milk for $1 \mathrm{~h}$, the membranes were probed with primary antibodies at $4^{\circ} \mathrm{C}$ overnight: antiTP53 (1:1000) and LC3 (1:500) were purchased from Cell Signaling Technology (CST; Danvers, MA, United States), while anti-p-AKT ${ }^{\operatorname{Ser} 473}$ (1:500), anti-p-mTOR (1:500), antip-P70S6K, anti-p-4EBP1, and P62 (1:500) were purchased from Sigma (St. Louis, MO, United States), and anti-GAPDH (1:5000) was purchased from CST, which served as a loading control. Then, the membrane was incubated with a secondary antibody for $2 \mathrm{~h}$. The protein expression was visualized using enhanced chemiluminescence (ECL) reagent (Thermo Fisher Scientific), and the concentration of proteins was quantified by Image J software.

\section{Cell Proliferation Assay}

Cell proliferation was analyzed using the crystal violet assay. Briefly, $5 \times 10^{5}$ stably transfected cells were seeded into each well of a six-well plate in duplicates and cultured for 1-4 days. Then, the cells were fixed with pre-cooled $10 \%$ formaldehyde for $20 \mathrm{~min}$ and stained with $0.1 \%$ crystal violet for $15 \mathrm{~min}$. The reaction was stopped by $10 \%$ glacial acetic acid, and the absorbance $(450 \mathrm{~nm})$ was measured on a SpectraMax Paradigm microplate reader (Molecular Devices, Sunnyvale, CA, United States).

\section{Cell Migration Test}

For the Transwell migration assay, $3 \times 10^{5}$ cells suspended in medium without serum were added to the upper chamber of the Transwell plates (Corning Inc., Corning, NY, United States), while $20 \%$ FBS-medium was added to the lower chamber. After incubation for $24 \mathrm{~h}$, the cells in the upper chamber were removed by a cotton swab, while the migrated cells attached to the bottom were fixed with $10 \%$ formaldehyde and stained with $0.1 \%$ crystal violet. Images were captures using a camera equipped with a BX41 Olympus microscope (Leica, Wetzlar, Germany). Three independent experiments were performed.

\section{Gene Set Enrichment Analysis of TP53 in Esophageal Cancer}

Gene set enrichment analysis is an analysis method for genomewide expression of the microarray data that compares the genes with predefined gene sets. Herein, we used the GSEA approach to analyze the biological functions of TP53 gene with 81 ESCC tissues obtained from TCGA using the ClusterProfiler and ggplot2 package in the $\mathrm{R}$ software. Using $|\mathrm{NES}|>1$ and $p$-value $<0.05$ as the threshold of GSEA, pathways were considered significantly enriched when they fulfilled the subconditions.

\section{Statistical Analysis}

The two sets of data of cancer tissues and adjacent tissue mRNA expression are skewness distribution, as analyzed by the non-parametric statistical method Wilcoxon test. The difference between TP53 expression and clinicopathologic characteristics was analyzed by Fisher's exact test. The data in this study were analyzed by GraphPad Prism 7.0 software (GraphPad, San Diego CA, United States) and the $\mathrm{R}$ software. $p<0.05$ indicates a statistically significant difference.

\section{RESULTS}

\section{High Transcriptional Expression of TP53 Family Members in Esophageal Cancer Patients}

To explore the transcriptional expression of TP53 family members in ESCA, we analyzed 33 types of cancers tissues and the corresponding normal tissues using the TIMER database. As shown in Figure 1A, the mRNA expression of TP53, TP63, and TP73 was significantly upregulated in ESCA tissues compared to normal tissues. Moreover, the comparison of the expression of TP53 family members based on the TCGA database showed a significantly higher expression of all TP53 members in ESCA tissues than normal specimens (Figures 1B-D). 


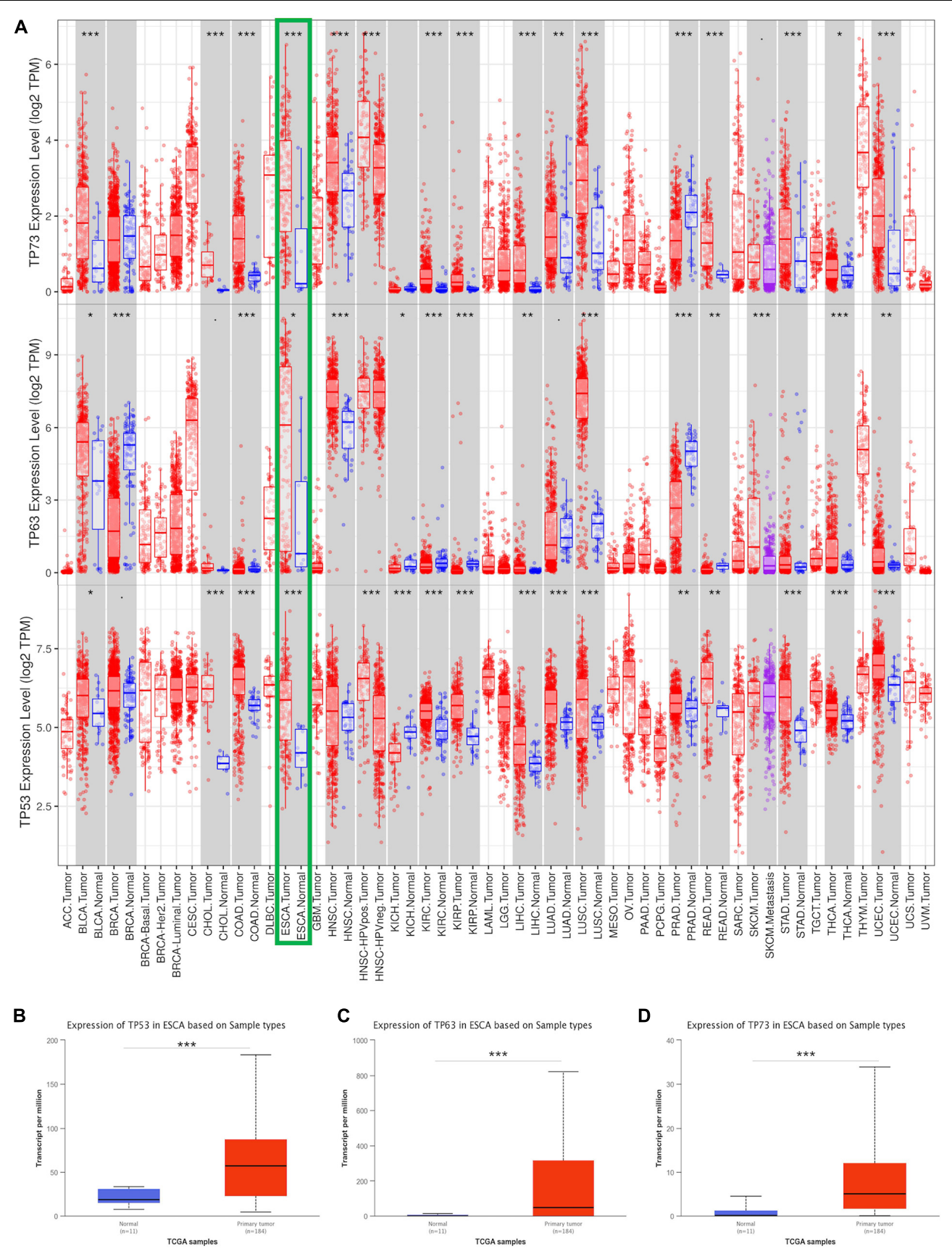

FIGURE 1 | Transcriptional expression of TP53 family member analysis. The mRNA levels of TP53 family members in 33 types of cancers from the TIMER database (A). The expression of TP53 family members between ESCA and non-cancerous tissues from the TCGA (B-D). * indicates $p<0.05$, ${ }^{* *}$ indicates $p<0.01$, ${ }^{\star \star \star}$ indicates $p<0.001$.

\section{Association of Transcriptional Expression of TP53 Family Members and the Clinical Parameters in Esophageal Cancer Patients}

The investigation of the association between clinicopathological features and mRNA expression of the TP53 family by UALCAN showed that the mRNA expressions of TP53 family members were significantly and positively associated with cancer stages, tumor grade, node metastasis, and tumor histology. Compared to non-cancerous tissues, the upregulated expression of TP53 family members was correlated with stage I, II, III, and IV (Figures 2A-C), while no statistically significant difference was detected in TP63 and TP73 mRNA expression between stages II and IV compared to non-cancerous tissues. However, the expression of TP53 family members only between tumor 
A

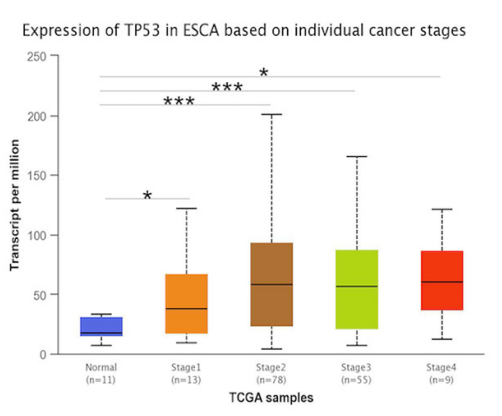

D

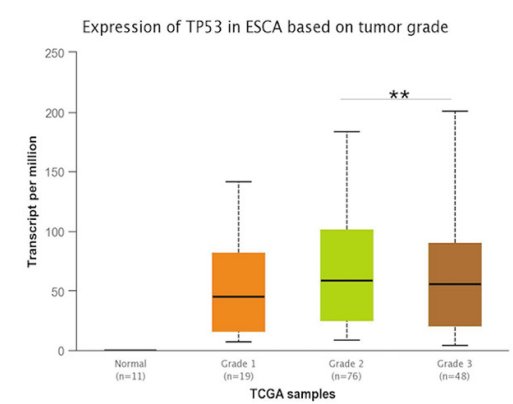

G

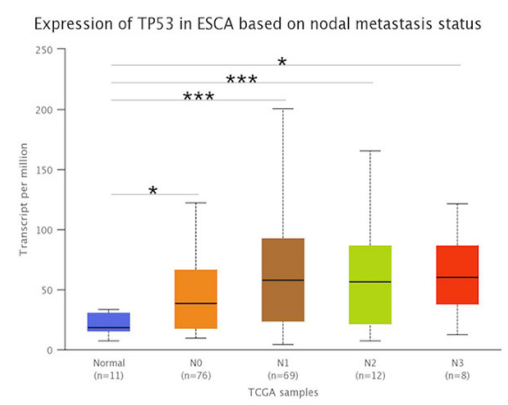

$\mathbf{J}$

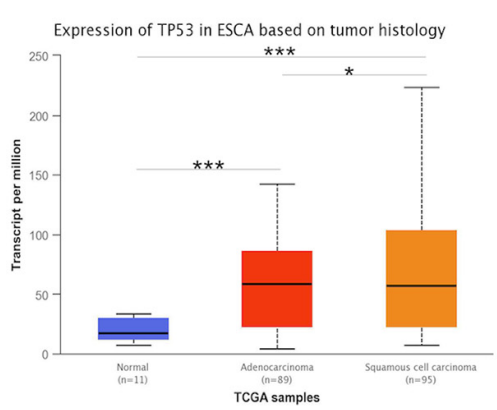

B

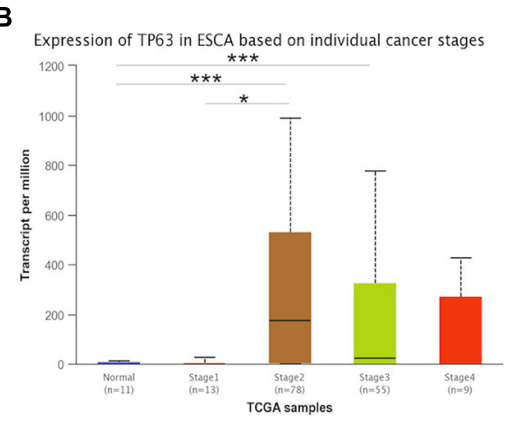

E

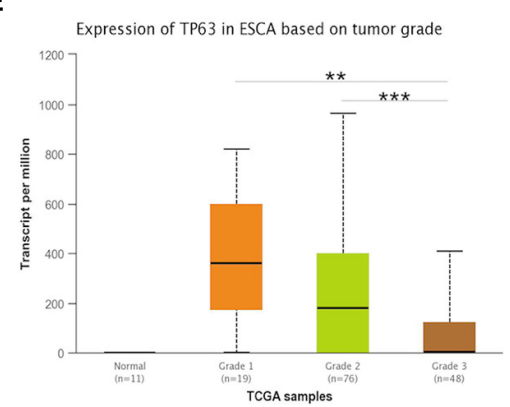

H

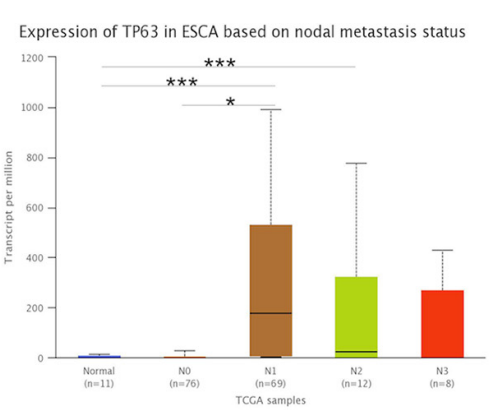

K

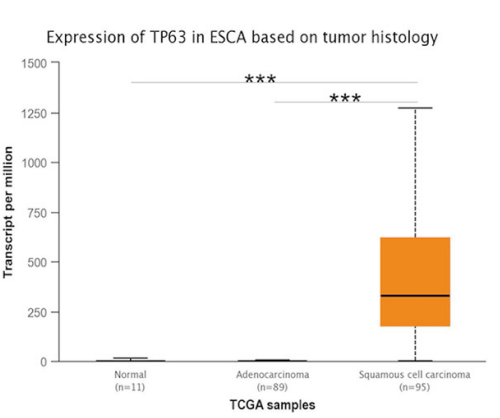

C

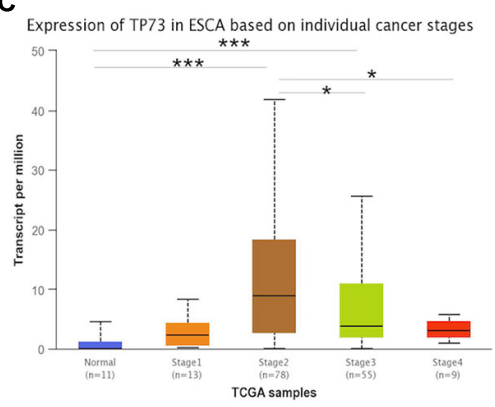

$\mathbf{F}$
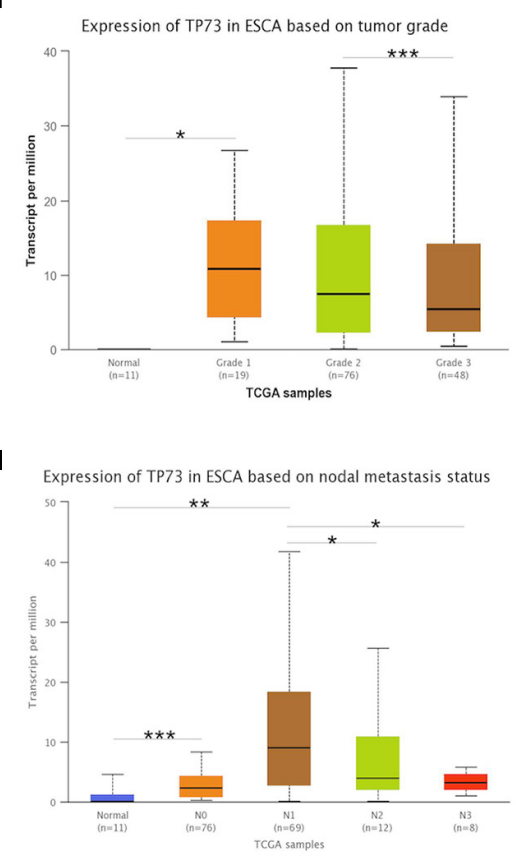

$\mathbf{L}$

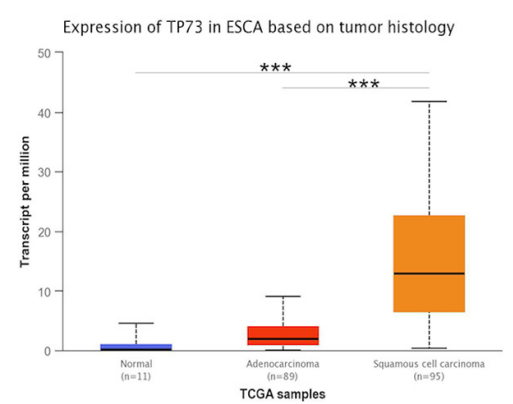

FIGURE 2 | Association between transcriptional expressions of TP53 family members and clinical parameters. The transcriptional expressions of TP53 family members associated with cancer stages, tumor grade, nodal metastasis status, and tumor histology (A-L). * indicates $p<0.05$, ** indicates $p<0.01$, and ${ }^{* \star *}$ indicates $p<0.001$.

grade 2 and grade 3 had obvious statistic difference. and TP73 mRNA expression in tumor grade 1 was significantly higher than non-cancerous tissues (Figures 2D-F).

Next, the association of the expression of TP53 family members to the node metastasis of ESCA patients was analyzed. As shown in Figure 2G, the mRNA expression of TP53 was positively associated with the node metastasis of ESCA patients. However, the expression of TP63 and TP73 did not differ significantly with nodal metastasis status in $\mathrm{N} 0, \mathrm{~N} 3$ and $\mathrm{N} 2$, and N3, respectively (Figures 2H,I).

We also analyzed the association between mRNA expressions of TP53 family members and the tumor histology of ESCA 
patients. As shown in Figures 2J-L, the mRNA expression of TP53 family members was significantly upregulated in ESCC tissues compared to normal tissues, while that of TP63 and TP73 did not show any remarkable difference in ECA tissues. Taken together, the mRNA expression of TP53 was significantly associated with the clinicopathological parameters of ESCA patients.

\section{Immune Infiltration in Correlation With Expression of TP53 Family Members in Esophageal Cancer Patients}

The correlation between the mRNA expression of TP53 family members with immune infiltration levels in ESCA was investigated using the TIMER database. The mRNA expression of TP53 was obviously related to tumor purity and neutrophils as shown in Figure 3A. However, B cells, CD8 + T cells, and dendritic cells were weakly not significantly correlated with the mRNA expression of TP63 (Figure 3B). On the other hand, the mRNA expression of TP73 showed a remarkable correlation with infiltrating levels of $\mathrm{B}$ cell and CD8 $+\mathrm{T}$ cells in ESCA (Figure 3C). The above data illustrated that TP53 family members were remarkably related to the infiltration of immune cells, indicating significant effects of TP53 family members on ESCA.

\section{Prognostic Value of TP53 Family Members in Esophageal Cancer Patients}

The prognostic value of TP53 family members in ESCA patients was analyzed by Kaplan-Meier plotter. As shown in Figure 4, TP53 [hazard ratio $(\mathrm{HR})=0.28$ and $\log$-rank $p=0.026$ ] showed a significantly good prognosis in ESCC patients when mRNA expression was upregulated, and TP73 ( $\mathrm{HR}=2.74$ and logrank $p=0.0043$ ) showed a negative correlation between high mRNA expression and significantly positive OS in EAC patients. However, the mRNA expression of TP63 did not show any statistically significant association with the prognosis of both ESCC and EAC patients. These results might provide additional evidence about the prognostic biomarkers for ESCA. Thus, we focused on the role of the TP53 in subsequent experiments.

\section{Verification mRNA Expression of TP53 in Esophageal Squamous Cell Carcinoma Patients}

Furthermore, we verified the mRNA expression of TP53 in 65 ESCC patients by RT-qPCR. The results of expression in ESCC patient tissues and the corresponding adjacent tissues were consistent with the analysis using the TCGA database, wherein the mRNA expression of TP53 was upregulated in ESCC tissues compared to the paired adjacent normal tissues (Figure 5A). Notably, $>90 \%$ (59/65) of ESCC tissues expressed high levels of TP53, while only $10 \%$ (6/65) of matched normal tissues expressed high levels of TP53 (Figure 5B). These findings strongly validated that the mRNA expression of TP53 is upregulated in ESCC.

The association between mRNA expression of TP53 and the clinical factors in 65 ESCC patients was analyzed. As shown in Table 1, high mRNA expression of TP53 in ESCC was positively correlated with TNM stage $(p=0.007)$ and lymph node metastasis $(p=0.030)$ in ESCC patients, while no association was observed with other clinicopathological features, including age, gender, and differentiation. Conversely, the correlation between the expression of TP53 and TNM stage and lymph node metastasis was consistent with the analysis using the TCGA database.

\section{TP53 Regulates the Proliferation and Migration of Esophageal Squamous Cell Carcinoma Cells}

To further investigate the potential biological function of TP53, the expression of TP53 in Kyse150 and TE1 ESCC cells was knocked down by stable transfection with sh-TP53 plasmids, while sh-NC plasmid was transfected in ESCC cells as a control. As shown in Figures 6A,B, the mRNA expression and the protein level of TP53 were downregulated by sh-TP53 in ESCC cells, which in turn significantly reduced the growth rate of Kyse150 and TE1 cells, as assessed by cell proliferation assay (Figures 6C,D). The Transwell assay showed that the downregulated expression of TP53 suppressed the migratory ability of ESCC cells (Figure 6E); the wound healing assay also displayed a similar trend (Figure 6F). Together, these findings indicated that TP53 is involved in the regulation of proliferation and migration of ESCC cells.

\section{TP53 Regulates mTOR Signaling Pathway in Esophageal Squamous Cell Carcinoma Cells}

The above results indicated that TP53 gene plays a vital role in tumorigenesis of ESCC. GSEA was utilized to describe the TP53 gene on the cellular process to investigate the underlying biological function. As shown in Supplementary Table 1, 27 gene sets were significantly enriched; among these, TP53 signaling pathway, mTOR signaling pathway, NOTCH signaling pathway, the mitogen-activated protein kinase (MAPK) signaling pathway, and pathway in cancer cell cycle are closely related to tumorigenesis. The enriched gene sets of TP53 and AKT-mTOR were shown by normalized enrichment score value ranking in Figures $\mathbf{7 A}, \mathbf{B}$. These findings indicated that TP53 promotes ESCC development by regulating AKT-mTOR signaling pathway. The investigation of AKT-mTOR signaling pathway by Western blotting in stably transfected sh-TP53 ESCC cells elucidated the molecular mechanisms underlying TP53 in tumorigenesis and development of ESCC. As shown in Figure 7C, the expression of TP53 was significantly decreased when transfected with sh-TP53 cells compared to sh-NC cells. The phosphorylation of p-p70S6K and $\mathrm{p}-4 \mathrm{EBP} 1$ was increased in TP53-downregulated ESCC cells, indicating that the downregulation of TP53 significantly enhanced the activation of AKT-mTOR pathway. Also, P62 and LC3-II also showed a declining trend compared to the sh- $N C$ group. Collectively, these results suggested that the downregulation of TP53 activates the transduction pathway of AKT-mTOR and inhibits autophagy, which may be responsible for its tumor-suppressive function. 

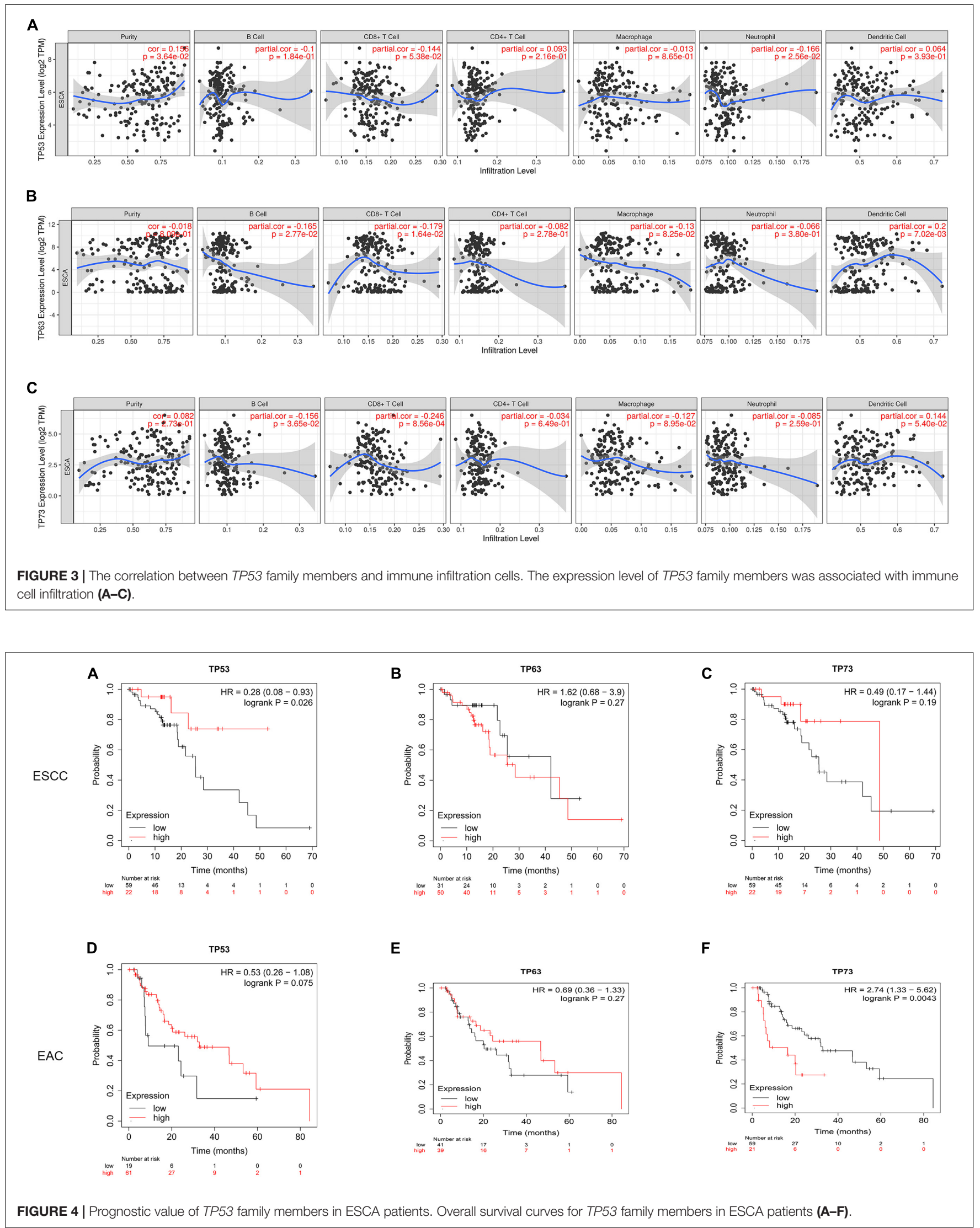
A

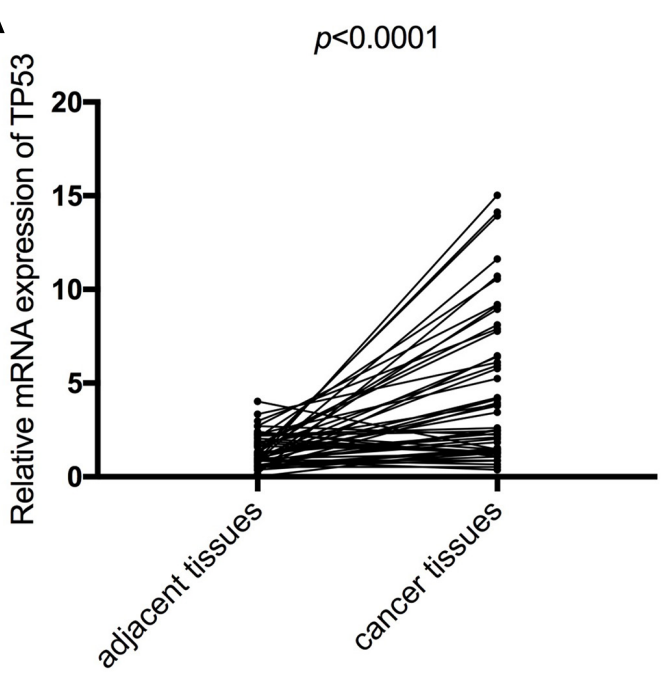

B

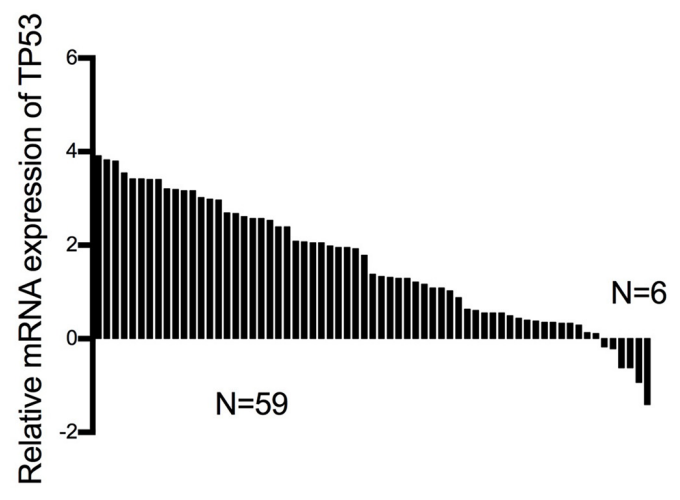

FIGURE 5 | The mRNA expression of TP53 validated in 65 patients with ESCC. The mRNA expression of TP53 and the matched adjacent tissues detected by qRT-PCR. Three independent experiments were conducted (A). High mRNA expression of TP53 more than 90\% (59/65) of ESCC tissues compared with adjacent normal tissues (B).

\section{DISCUSSION}

Various molecules are known to be associated with the development and progression of ESCA. TP53 family members are

TABLE 1 | Association of clinicopathological data and expression of TP53 in ESCC patients.

\begin{tabular}{|c|c|c|c|c|}
\hline \multirow[t]{2}{*}{ Patient characteristics } & \multicolumn{2}{|c|}{ TP53 expression } & \multirow[t]{2}{*}{$x^{2}$} & \multirow[t]{2}{*}{$P$-value } \\
\hline & High $(n=59)$ & Low $(n=6)$ & & \\
\hline Age & & & 0.437 & 0.508 \\
\hline$<65$ & 28 & 2 & & \\
\hline$\geq 65$ & 31 & 4 & & \\
\hline Gender & & & 0.271 & 0.603 \\
\hline Male & 45 & 4 & & \\
\hline Female & 14 & 2 & & \\
\hline Tumor size & & & 0.538 & 0.764 \\
\hline$<3 \mathrm{~cm}$ & 15 & 2 & & \\
\hline $3-5 \mathrm{~cm}$ & 40 & 4 & & \\
\hline$>5 \mathrm{~cm}$ & 4 & 0 & & \\
\hline Tumor location & & & 1.06 & 0.589 \\
\hline Upper & 9 & 1 & & \\
\hline Middle & 40 & 3 & & \\
\hline lower & 10 & 2 & & \\
\hline TNM stage & & & 12.2 & $0.007^{*}$ \\
\hline I & 7 & 4 & & \\
\hline ॥ & 31 & 2 & & \\
\hline III & 19 & 0 & & \\
\hline IV & 2 & 0 & & \\
\hline Lymph node metastasis & & & 4.697 & $0.030^{*}$ \\
\hline Yes & 27 & 0 & & \\
\hline No & 32 & 6 & & \\
\hline Differention & & & 1.036 & 0.596 \\
\hline Well & 20 & 1 & & \\
\hline Moderate & 27 & 4 & & \\
\hline Poor & 12 & 1 & & \\
\hline
\end{tabular}

Using a Fisher's exact test. The $p$-value was set at 0.05 and ${ }^{*}$ indicates $p<0.05$. known as tumor suppressors participate in the development of several tumors. The TP53 family members and their interactions with other factors promoted progression of thyroid cancer (Manzella et al., 2017). The correlation between the TP53 family members and the Notch signaling pathway is often inactivated in the cutaneous squamous cell carcinoma (Missero and Antonini, 2014). All TP53 family members are abnormally expressed in bladder cancer, and TP73 overexpression is associated with aggressive tumor phenotype (Papadogianni et al., 2014). The immunostaining in oral squamous cell carcinomas revealed that the TP53 family members are biomarkers that could contribute to the diagnosis and monitoring of high-risk precancerous lesions of the oral epithelium (Bidaud et al., 2010). The abnormal expression of TP63 and TP73 is associated with hematological malignancy grade and poor prognosis (Alexandrova and Moll, 2012). TP53 family members constitute an interacting network involved in cellular responses to chemotherapeutic drugs in gastrointestinal cancer (Vilgelm et al., 2010). Moreover, a differential expression of TP53 family members is observed in the antimitotic agent vincristine-treated TP53-deficient breast cancer cells (Vayssade et al., 2002). However, the function and prognosis of TP53 family members in ESCA are yet unknown. To the best of our knowledge, our study is the first clarifying the systematic analysis of TP53 family genes in ESCA patients using multiple bioinformatics databases. The results showed that mRNA expression of TP53, TP63, and TP73 in ESCA tissues was significantly higher than in normal tissues. Moreover, the expression of TP53 was upregulated in both ESCC and EAC, while high expression of TP63 and TP73 was observed only in EAC. Furthermore, we investigated the association between the clinicopathological data and the expression of TP53 family members of ESCA patients. The mRNA expression of TP53 was remarkably correlated with cancer stages (stage I, II, III, and IV) and nodal metastasis status in ESCA tissues, while the expression 
A

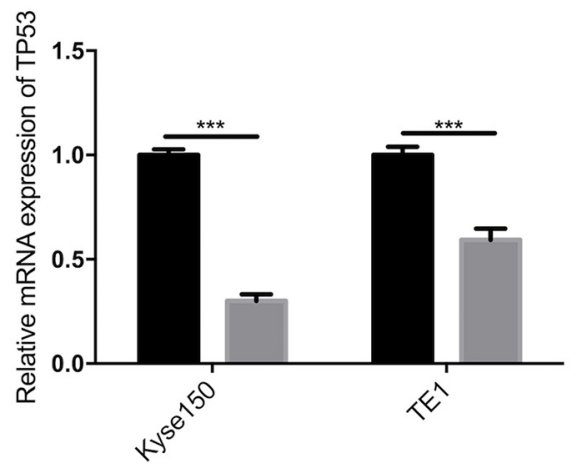

C

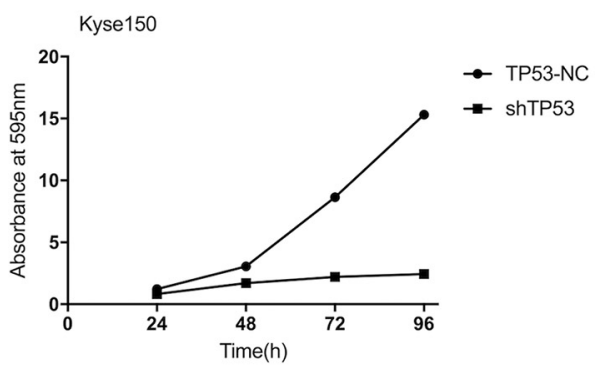

D

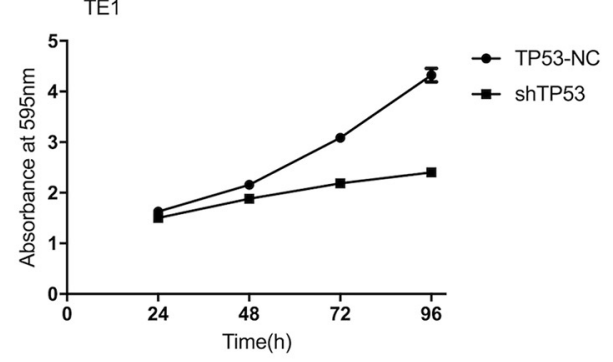

\section{B}

TP53-NC

shTP53

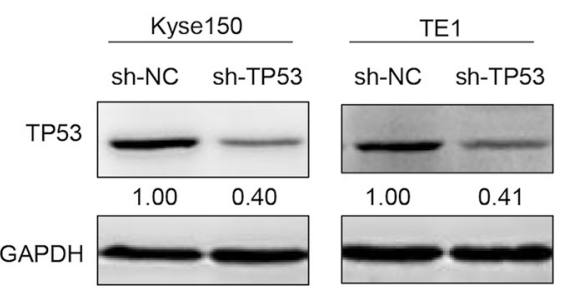

E

sh-NC
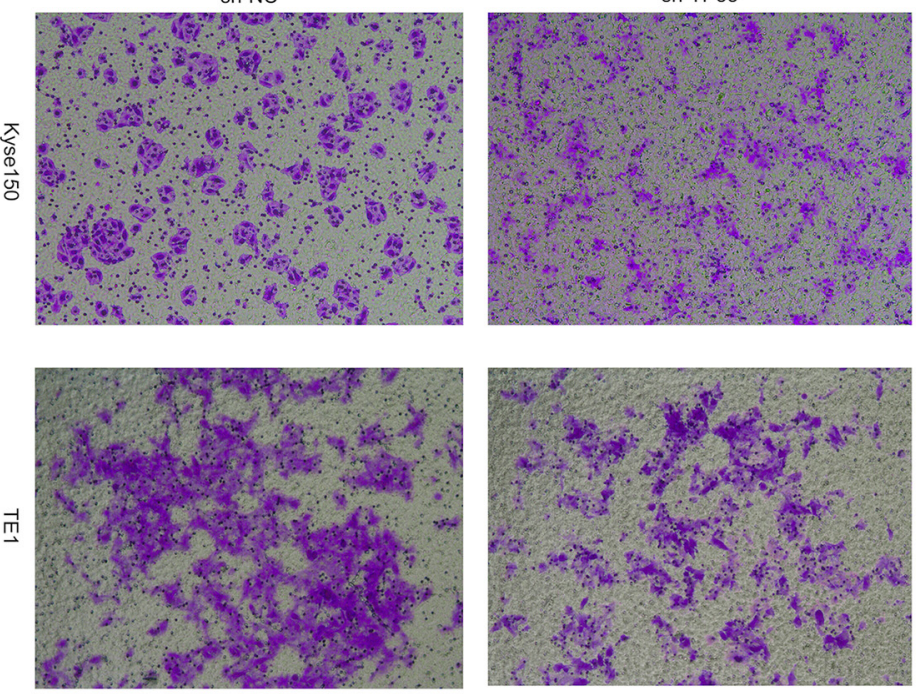

F
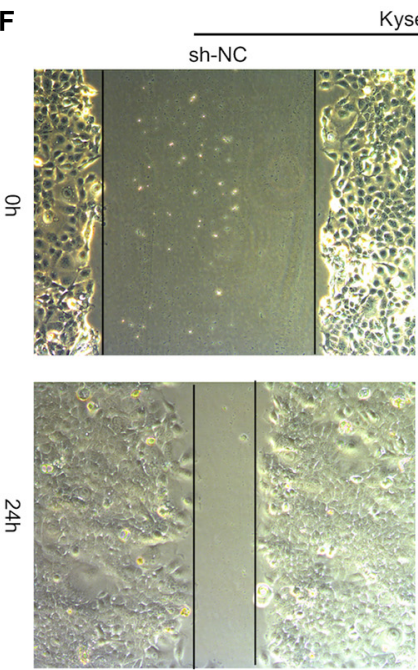

Kyse150
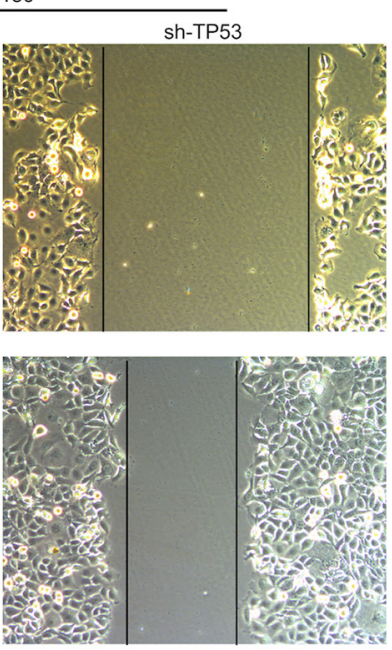
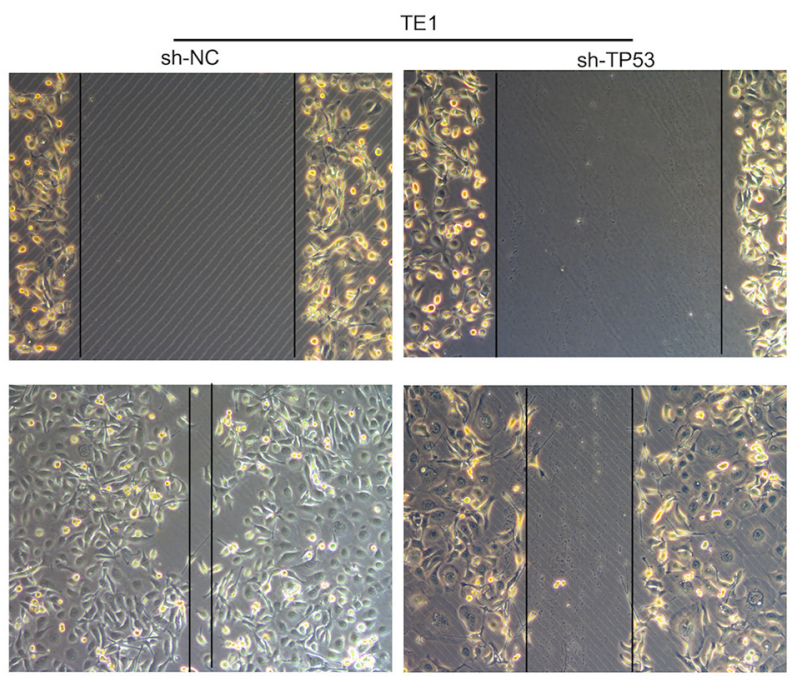

FIGURE 6 | TP53 regulates the proliferation and migration of ESCC cells. KYSE-150 and TE1 cells were stably transfected with plasmids (sh-NC and sh-TP53). The mRNA expression and protein expression of TP53 were determined by qRT-PCR and Western blotting (A,B). Crystal violet assay demonstrated that knockdown of TP53 significantly inhibited cell proliferation (C,D). Transwell and Scratch test showed downregulated expression of TP53 decreased cell migration (E,F). Data are presented as mean $\pm \mathrm{SD}$; ${ }^{* \star \star}$ indicates $p<0.001$. 


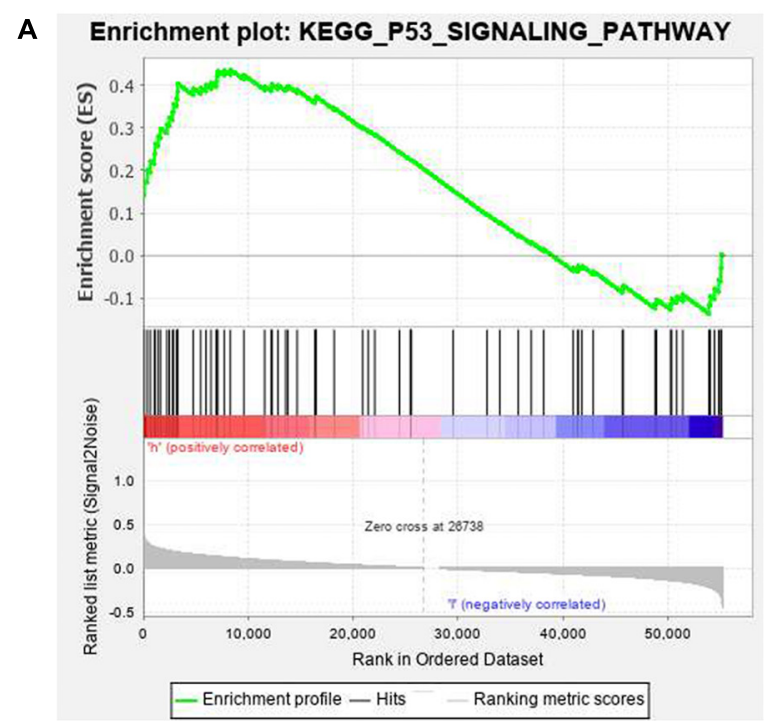

B Enrichment plot: KEGG_MTOR_SIGNALING_PATHWAY

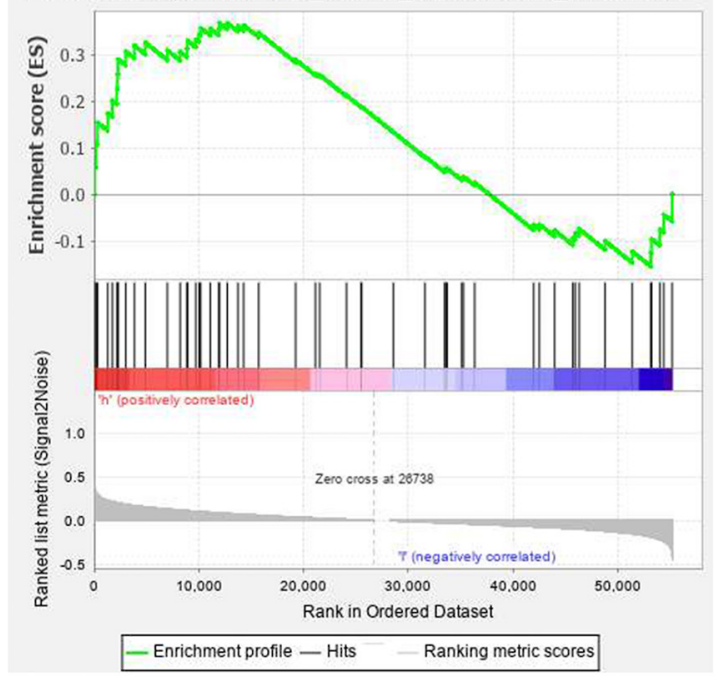

C

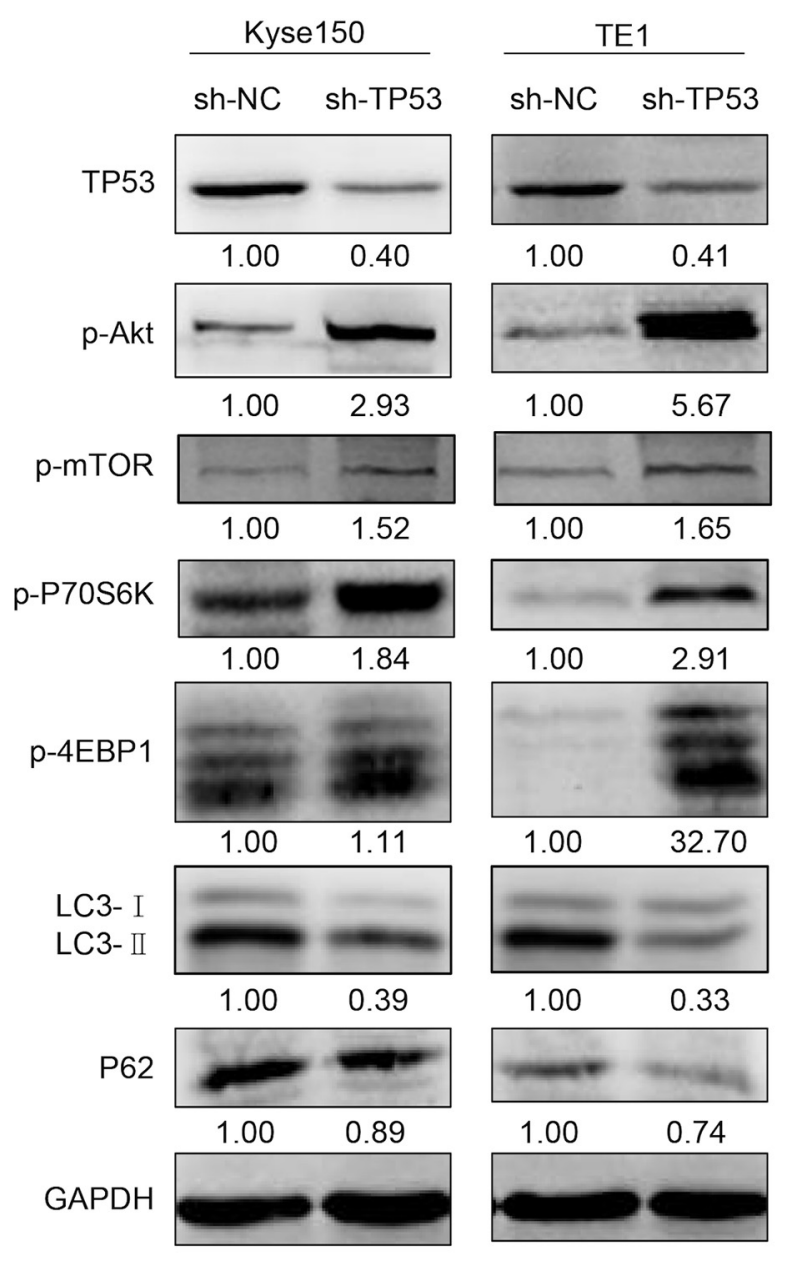

FIGURE 7 | TP53 regulated mTOR signaling pathway in ESCC cells. GSEA enrichment plots demonstrated that TP53 was positively correlated with the TP53 signaling pathway and mTOR signaling pathway (A,B). Western blotting analysis of the mTOR signaling pathway-and autophagy-associated proteins (C).

of TP63 and TP73 was correlated with cancer stages II and III. The study also displayed the lack of correlation between mRNA expression of the TP53 family and tumor grade.

To address the expression and function of TP53 in ESCA, we detected the level of TP53 in ESCA tissue. Owing to the high morbidity rate of ESCC in China, we selected ESCC cell lines (Kyse150 and TE1) for further study. TP53 is a tumorsuppressor gene but is mutated in about $50 \%$ of cancers, thereby regulating the proliferation of various tumor cells (Egashira et al., 2011). A high expression of TP53 has been observed in many malignancies (Hinds et al., 1990; Iggo et al., 1990; Samuels-Lev et al., 2001), including esophageal cancer (Huang et al., 2014; Yao et al., 2014; Xie et al., 2017). Several studies found that the expression of TP53 in cancer tissues of ESCC patients could be utilized to analyze the survival and prognosis of esophageal cancer (Yao et al., 2014; Xie et al., 2017; Melling et al., 2019).
These findings strongly suggested that TP53 is involved in the tumorigenesis of ESCA. Herein, we also verified that the mRNA expression of TP53 was remarkably upregulated in ESCC tissues. Also, the analysis of clinicopathological parameters showed that TP53 levels were positively correlated with TNM stage and lymph node metastasis. In addition, Kaplan-Meier analysis revealed that overexpression of TP53 in ESCC patients is correlated with prognosis. The downregulated expression of TP53 significantly suppressed the ESCC cell growth and migration. These findings revealed that TP53 exerts an oncogenic role in the initiation and progression of ESCC.

Previous studies have also demonstrated the role of TP53 in immune response and tumor microenvironment. Blagih and Levine reported that TP53 regulates immune cells to participate in B-cell and CD8 killer T-cell response of cancer cells (Blagih et al., 2020; Levine, 2020a). Vadakekolathu et al. (2020) found 
that TP53 abnormalities are involved in immune response and beneficial for immunotherapy of acute myeloid leukemia. Based on the TCGA-ESCA dataset, we found that TP53 was significantly correlated with the abundance of immune cells. Our results might imply that TP53 may carry out an immune escape role in the ESCA microenvironment.

In order to further explore the molecular mechanism of TP53 regulating the proliferation and migration in ESCC cells, we analyzed the effects of TP53 gene on the cellular process using the GSEA approach. The results suggested that TP53 and mTOR signaling pathways promote ESCC progression by influencing the pathways in cancer. Subsequently, we demonstrated that the activation of AKT-mTOR pathway was significantly enhanced in TP53 downregulated ESCC cells. In terms of molecular mechanisms, TP53 regulates cell cycle and apoptosis and protects the cells from DNA-damaging agents (Case and Domann, 2014; de Assis and Isoldi, 2014). In addition, TP53 is also involved in other functions, such as immune response, senescence, cellular differentiation, angiogenesis, DNA metabolism, and senescence (Suzuki and Matsubara, 2011). Additionally, TP53 mediates autophagy in some pathological factors. Feng et al. (2005) found that the induction of TP53 by DNA damaging agents inhibited mTOR and induced autophagy in mouse embryo fibroblasts. Budanov and Karin (2008) also demonstrated that Sestrin1 and Sestrin 2 are the products of two TP53 target genes, which inhibit mTOR via binding and activating AMPK. Importantly, loss of Sestrin2 significantly reduced TP53-mediated autophagy. The combined data indicated that TP53 inhibits the mTOR pathway and subsequently induces autophagy. The mTOR pathway plays a vital role in the regulation of cell growth and proliferation (Hay and Sonenberg, 2004). In mammals, mTOR is regulated by PI3K, PI3K-dependent kinase 1, and AKT. Subsequently, mTOR regulates cellular processes by regulating its downstream targets including p70S6 kinase and eIF4E binding protein 1 (4EBP1), which are critical regulators of translation (Brown et al., 1995; Brunn et al., 1997). In this study, the phosphorylation of AKT, mTOR, 70S6K, and 4EBP1 was increased in TP53-downregulated ESCC cells. Furthermore, P62 and LC3-II showed a declining trend in TP53-knockdown cells. These findings indicated that the downregulation of TP53 expression mediates cell translation via MTOR pathway. Moreover, TP53 downexpression activates mTOR and inhibits autophagy. Thus, it could be speculated that TP53 mediates the mTOR pathway to regulate the proliferation and autophagy in ESCC cells. However, these findings need to be validated clinically.

\section{CONCLUSION}

This study showed that TP53 is upregulated in ESCC tissues and plays a vital role in ESCC cell proliferation and migration. It also

\section{REFERENCES}

Abnet, C. C., Arnold, M., and Wei, W. Q. (2018). Epidemiology of esophageal squamous cell carcinoma. Gastroenterology 154, 360-373. doi: 10.1053/j.gastro. 2017.08.023 might activate the mTOR signaling pathway and inhibit TP53dependent autophagy. Thus, this might provide new insights into the potential role of TP53 as a diagnostic biomarker and valuable therapeutic target in ESCC.

\section{DATA AVAILABILITY STATEMENT}

The datasets presented in this study can be found in online repositories. The names of the repository/repositories and accession number(s) can be found in the article/ Supplementary Material.

\section{ETHICS STATEMENT}

The studies involving human participants were reviewed and approved by the Medical Ethics Committee of the Affiliated Hospital of North Sichuan Medical College. The patients/participants provided their written informed consent to participate in this study.

\section{AUTHOR CONTRIBUTIONS}

XG, LY, and XZ conceived and designed the experiments. LY and $\mathrm{XZ}$ performed the experiments and wrote the initial draft of the manuscript. GH and QM contributed to the statistical analysis. $\mathrm{GH}$ contributed to the clinical data collection. LX and HX were involved in the provision of study materials. XG reviewed and edited the manuscript. All authors reviewed and approved the final manuscript.

\section{FUNDING}

This research was supported by the Applied Basic Research Program of Sichuan Province (Grant No. 2021YJ0202), the Science and Technology Support Program of Nanchong (Grant Nos. 19SXHZ0244 and 18SXHZ0514), and the Development of Scientific Research Plan of Doctoral Scientific Research Foundation of North Sichuan Medical College (Grant Nos. 2020JC031 and 2020ZD022).

\section{SUPPLEMENTARY MATERIAL}

The Supplementary Material for this article can be found online at: https://www.frontiersin.org/articles/10.3389/fcell.2021. 730337/full\#supplementary-material

Supplementary Table 1 | GSEA of pathways significantly represented in ESCA.

Agostini, M., Melino, G., and Bernassola, F. (2018). The p53 family in brain disease. Antioxid. Redox Signal. 29, 1-14. doi: 10.1089/ars.2017.7302

Alexandrova, E. M., and Moll, U. M. (2012). Role of p53 family members p73 and p63 in human hematological malignancies. Leuk. Lymphoma 53, 2116-2129. doi: $10.3109 / 10428194.2012 .684348$ 
Arnold, M., Soerjomataram, I., Ferlay, J., and Forman, D. (2015). Global incidence of oesophageal cancer by histological subtype in 2012. Gut 64, 381-387. doi: 10.1136/gutjnl-2014-308124

Bidaud, P., Chasle, J., Sichel, F., Rousseau, S., Petit, P., Pottier, D., et al. (2010). Expression of p53 family members and CD44 in oral squamous cell carcinoma (OSCC) in relation to tumorigenesis. Histol. Histopathol. 25, 331-339.

Bieging, K. T., Mello, S. S., and Attardi, L. D. (2014). Unravelling mechanisms of p53-mediated tumour suppression. Nat. Rev. Cancer 14, 359-370. doi: 10.1038/ nrc3711

Blagih, J., Buck, M. D., and Vousden, K. H. (2020). p53, cancer and the immune response. J. Cell. Sci. 133:jcs237453. doi: 10.1242/jcs.237453

Brown, E. J., Beal, P. A., Keith, C. T., Chen, J., Shin, T. B., and Schreiber, S. L. (1995). Control of p70 s6 kinase by kinase activity of FRAP in vivo. Nature 377, 441-446. doi: 10.1038/377441a0

Brunn, G. J., Hudson, C. C., Sekulic, A., Williams, J. M., Hosoi, H., Houghton, P. J., et al. (1997). Phosphorylation of the translational repressor PHAS-I by the mammalian target of rapamycin. Science 277, 99-101. doi: 10.1126/science.277. 5322.99

Budanov, A. V., and Karin, M. (2008). p53 target genes sestrin1 and sestrin2 connect genotoxic stress and mTOR signaling. Cell 134, 451-460. doi: 10.1016/ j.cell.2008.06.028

Bykov, V. J. N., Eriksson, S. E., Bianchi, J., and Wiman, K. G. (2018). Targeting mutant p53 for efficient cancer therapy. Nat. Rev. Cancer 18, 89-102. doi: 10.1038/nrc.2017.109

Case, A. J., and Domann, F. E. (2014). Absence of manganese superoxide dismutase delays p53-induced tumor formation. Redox Biol. 2, 220-223. doi: 10.1016/j. redox.2014.01.001

Chen, G., Wang, Z., Liu, X. Y., and Liu, F. Y. (2007). Recurrence pattern of squamous cell carcinoma in the middle thoracic esophagus after modified IvorLewis esophagectomy. World J. Surg. 31, 1107-1114. doi: 10.1007/s00268-0060551-1

Chen, J., Kwong, D. L., Cao, T., Hu, Q., Zhang, L., Ming, X., et al. (2015). Esophageal squamous cell carcinoma (ESCC): advance in genomics and molecular genetics. Dis. Esophagus 28, 84-89. doi: 10.1111/dote.12088

Chen, W., Zheng, R., Zeng, H., and Zhang, S. (2015). The updated incidences and mortalities of major cancers in China, 2011. Chin. J. Cancer 34, 502-507. doi: 10.1186/s40880-015-0042-6

de Assis, L. V., and Isoldi, M. C. (2014). The function, mechanisms, and role of the genes PTEN and TP53 and the effects of asbestos in the development of malignant mesothelioma: a review focused on the genes' molecular mechanisms. Tumour Biol. 35, 889-901. doi: 10.1007/s13277-013-1210-4

Dubecz, A., Gall, I., Solymosi, N., Schweigert, M., Peters, J. H., Feith, M., et al. (2012). Temporal trends in long-term survival and cure rates in esophageal cancer: a SEER database analysis. J. Thorac. Oncol. 7, 443-447. doi: 10.1097/ JTO.0b013e3182397751

Egashira, A., Morita, M., Yoshida, R., Saeki, H., Oki, E., Sadanaga, N., et al. (2011). Loss of p53 in esophageal squamous cell carcinoma and the correlation with survival: analyses of gene mutations, protein expression, and loss of heterozygosity in Japanese patients. J. Surg. Oncol. 104, 169-175. doi: 10.1002/ jso. 21920

Feng, Z., Zhang, H., Levine, A. J., and Jin, S. (2005). The coordinate regulation of the p53 and mTOR pathways in cells. Proc. Natl. Acad. Sci. U.S.A. 102, 8204-8209. doi: 10.1073/pnas.0502857102

Ferlay, J., Soerjomataram, I., Ervik, M., Dikshit, R., Eser, S., and Mathers, C. (2013). GLOBOCAN 2012 v1.0, Cancer Incidence and Mortality Worldwide: IARC CancerBase No. 11. Lyon: International Agency for Research on Cancer.

Gao, Y. B., Chen, Z. L., Li, J. G., Hu, X. D., Shi, X. J., Sun, Z. M., et al. (2014). Genetic landscape of esophageal squamous cell carcinoma. Nat. Genet. 46, 1097-1102. doi: $10.1038 / \mathrm{ng} .3076$

Global Burden of Disease Cancer Collaboration, C., Dicker, D., Pain, A., Hamavid, H., Moradi-Lakeh, M., et al. (2015). The global burden of cancer 2013. JAMA Oncol. 1, 505-527.

Hay, N., and Sonenberg, N. (2004). Upstream and downstream of mTOR. Genes Dev. 18, 1926-1945. doi: 10.1101/gad.1212704

Hinds, P. W., Finlay, C. A., Quartin, R. S., Baker, S. J., Fearon, E. R., Vogelstein, B., et al. (1990). Mutant p53 DNA clones from human colon carcinomas cooperate with ras in transforming primary rat cells: a comparison of the "hot spot" mutant phenotypes. Cell Growth Differ. 1, 571-580.
Huang, K., Chen, L., Zhang, J., Wu, Z., Lan, L., Wang, L., et al. (2014). Elevated p53 expression levels correlate with tumor progression and poor prognosis in patients exhibiting esophageal squamous cell carcinoma. Oncol. Lett. 8, 1441-1446. doi: 10.3892/ol.2014.2343

Iggo, R., Gatter, K., Bartek, J., Lane, D., and Harris, A. L. (1990). Increased expression of mutant forms of p53 oncogene in primary lung cancer. Lancet 335, 675-679. doi: 10.1016/0140-6736(90)90801-B

Kandoth, C., McLellan, M. D., Vandin, F., Ye, K., Niu, B., Lu, C., et al. (2013). Mutational landscape and significance across 12 major cancer types. Nature 502, 333-339. doi: 10.1038/nature12634

Lane, D. P. (1992). Cancer. p53, guardian of the genome. Nature 358, 15-16. doi: $10.1038 / 358015 \mathrm{a} 0$

Levine, A. J. (2020b). p53: 800 million years of evolution and 40 years of discovery. Nat. Rev. Cancer 20, 471-480. doi: 10.1038/s41568-020-0262-1

Levine, A. J. (2020a). P53 and the immune response: 40 years of exploration-a plan for the future. Int. J. Mol. Sci. 21:541. doi: 10.3390/ijms21020541

Levrero, M., De Laurenzi, V., Costanzo, A., Gong, J., Wang, J. Y., and Melino, G. (2000). The p53/p63/p73 family of transcription factors: overlapping and distinct functions. J. Cell. Sci. 113(Pt 10), 1661-1670. doi: 10.1242/jcs.113.10. 1661

Lin, Y., Totsuka, Y., He, Y., Kikuchi, S., Qiao, Y., Ueda, J., et al. (2013). Epidemiology of esophageal cancer in Japan and China. J. Epidemiol. 23, 233-242. doi: 10.2188/jea.JE20120162

Manzella, L., Stella, S., Pennisi, M. S., Tirro, E., Massimino, M., Romano, C., et al. (2017). New insights in thyroid cancer and p53 family proteins. Int. J. Mol. Sci. 18:1325. doi: $10.3390 /$ ijms 18061325

Melling, N., Norrenbrock, S., Kluth, M., and Simon, R. (2019). p53 overexpression is a prognosticator of poor outcome in esophageal cancer. Oncol. Lett. 17, 3826-3834. doi: 10.3892/ol.2019.10020

Missero, C., and Antonini, D. (2014). Crosstalk among p53 family members in cutaneous carcinoma. Exp. Dermatol. 23, 143-146. doi: 10.1111/exd.12320

Oren, M., and Rotter, V. (2010). Mutant p53 gain-of-function in cancer. Cold Spring Harb. Perspect. Biol. 2:a001107. doi: 10.1101/cshperspect.a001107

Papadogianni, D., Soulitzis, N., Delakas, D., and Spandidos, D. A. (2014). Expression of p53 family genes in urinary bladder cancer: correlation with disease aggressiveness and recurrence. Tumour Biol. 35, 2481-2489. doi: 10. 1007/s13277-013-1328-4

Ryan, K. M. (2011). p53 and autophagy in cancer: guardian of the genome meets guardian of the proteome. Eur. J. Cancer 47, 44-50. doi: 10.1016/j.ejca.2010.10. 020

Samuels-Lev, Y., O’Connor, D. J., Bergamaschi, D., Trigiante, G., Hsieh, J. K., Zhong, S., et al. (2001). ASPP proteins specifically stimulate the apoptotic function of p53. Mol. Cell 8, 781-794. doi: 10.1016/S1097-2765(01)00367-7

Santos-Pereira, J. M., Gallardo-Fuentes, L., Neto, A., Acemel, R. D., and Tena, J. J. (2019). Pioneer and repressive functions of p63 during zebrafish embryonic ectoderm specification. Nat. Commun. 10:3049. doi: 10.1038/s41467-01911121-z

Suzuki, K., and Matsubara, H. (2011). Recent advances in p53 research and cancer treatment. J. Biomed. Biotechnol. 2011:978312. doi: 10.1155/2011/978312

Vadakekolathu, J., Lai, C., Reeder, S., Church, S. E., Hood, T., Lourdusamy, A., et al. (2020). TP53 abnormalities correlate with immune infiltration and associate with response to flotetuzumab immunotherapy in AML. Blood Adv. 4, 5011-5024. doi: 10.1182/bloodadvances.2020002512

Vayssade, M., Faridoni-Laurens, L., Benard, J., and Ahomadegbe, J. C. (2002). Expression of p53-family members and associated target molecules in breast cancer cell lines in response to vincristine treatment. Biochem. Pharmacol. 63, 1609-1617. doi: 10.1016/S0006-2952(02)00917-6

Vilgelm, A. E., Washington, M. K., Wei, J., Chen, H., Prassolov, V. S., and Zaika, A. I. (2010). Interactions of the p53 protein family in cellular stress response in gastrointestinal tumors. Mol. Cancer Ther. 9, 693-705. doi: 10.1158/1535-7163. MCT-09-0912

Vousden, K. H., and Prives, C. (2009). Blinded by the light: the growing complexity of p53. Cell 137, 413-431. doi: 10.1016/j.cell.2009.04.037

Xie, X.-F., Yang, Q., Chi, J., Yang, X.-Z., and Wang, H.-Y. (2017). Prognostic values of apoptosis-stimulating P53-binding protein 1 and 2 and their relationships with clinical characteristics of esophageal squamous cell carcinoma patients: a retrospective study. Chin. J. Cancer 36:15. doi: 10.1186/s40880-0160169-0 
Yao, W., Qin, X., Qi, B., Lu, J., Guo, L., Liu, F., et al. (2014). Association of p53 expression with prognosis in patients with esophageal squamous cell carcinoma. Int. J. Clin. Exp. Pathol. 7, 7158-7163.

Conflict of Interest: The authors declare that the research was conducted in the absence of any commercial or financial relationships that could be construed as a potential conflict of interest.

Publisher's Note: All claims expressed in this article are solely those of the authors and do not necessarily represent those of their affiliated organizations, or those of the publisher, the editors and the reviewers. Any product that may be evaluated in this article, or claim that may be made by its manufacturer, is not guaranteed or endorsed by the publisher.

Copyright (c) 2021 Yao, Zhong, Huang, Ma, Xu, Xiao and Guo. This is an open-access article distributed under the terms of the Creative Commons Attribution License (CC BY). The use, distribution or reproduction in other forums is permitted, provided the original author(s) and the copyright owner(s) are credited and that the original publication in this journal is cited, in accordance with accepted academic practice. No use, distribution or reproduction is permitted which does not comply with these terms. 